\title{
ECTOMICORRIZAS EN Nothofagus alpina (P.et E.) Oerst Y N. dombeyi (Mirb.) Oerst. DEL SUR DE CHILE
}

\author{
(Ectomycorrhizae in Nothofagus alpina (P.et E.) Oerst. and \\ N. dombeyi (Mirb.) Oerst. of Southern Chile)
}

\author{
Godoy, R. (*) \& G. Palfner (**) \\ * Instituto de Botánica, Universidad Austral de Chile, Casilla 567, Valdivia, Chile. \\ ** Institut für Systematische Botanik, Ludwig-Maximilians-Universität \\ München, Menzingerstrasse 67, D-80638 München, Alemania.
}

Palabras clave: Ectomicorrizas, simbiosis, Nothofagus alpina, N. dombeyi, Chile.

Key words: Ectomycorrhizac, symbiosis, Nothofagus alpina, $N$. dombeyi, Chile.

\section{RESUMEN}

Mediante estudios de microscopia óptica se describe y caracteriza la simbiosis ectomicorricica de Laccaria laccata (Scop) Berk + Nothofagus dombeyi (Mirb.) Oerst y Paxillus involutus (Batsch) Fr.+ Nothofagus alpina (P. et E.) Oerst, en plántulas obtenidas de vivero en la Provincia de Valdivia, Sur de Chile. Se documenta la micorriza mediante ilustración del hábito y microfotografias del manto fíngico, asi como de secciones transversales y longitudinales, . La estructura morfo-anatómica de las especies de hongos estudiadas, se compara con hospedantes de Europa. Se destaca la importancia de la asociación micorricica, para futuros programas de reforestación en el Sur de Chile

\section{INTRODUCCION}

Las simbiosis micorrícicas, frecuentes en las comunidades boscosas nativas del Sur de Chile (Singer \& Morello 1960, Gamundi \& Horak 1993, Godoy 'ct al. 1995), juegan un rol destacado, en lo que se refiere a la absorción y transporte de nutrientes y agua, a través de una estrecha red de hifas en el suclo, permitiendo el traspaso de sustancias a nivel intra e interspecífico, factor de extraordinaria importancia bajo condiciones limitantes del sustrato (Marx 1991). Contribuyen además. al agregado de las partículas del suelo, favoreciendo el rápido crecimiento y establecimiento de la regeneración natural (Perry \& Amaranthus 1990, Marx 1991).

El género Nothofagus, componentc importante en

\section{SUMMARY}

The ectomycorr hizae Laccaria laccata (Scop) Berk + Nothofagus dombeyi(Mirb.) Oerst and Paxillus involutus (Batsch) Frr. + Nothofagus alpina (P. et E.) Oerst, are described morphologically and anatomically on nursery seedlings from the province of Valdivia, southern Chile. The mycorrhizae are documented macroscopically and microscopically by photographs of the fungal mantle and emanating elements in plan view, transversal and longitudinal sections of whole mycorrhizal tips. The relationship to european mycorrhizae, formed by the same fingal species is discussed as well as the importance of the symbiosis for future reforestation programme in Southern Chile

los bosques del Sur de Chile (Kalin- Arroyo et al. 1995), presenta una extraordinaria dependencia por hongos simbiontes, estructurando la denominada ectomicorriza (Garrido 1988, Valcnzucla 1993, Palfner \& Godoy 1996, $\mathrm{a}, \mathrm{b})$.

Desafortunadamente, algunos registros de ectomicorrizas, son asignados por la presencia de cuerpos fructífcros juntos a los hospedantes y son designados como hongos micorrícicos putativos (Flores et al. 1997). Garrido (1988), indica 651 taxa de hongos micorrícicos, para el género Nothofagus en su distribución global, con un fucrte dominio en las especies de Chile, cuya comprobación y descripción de la asociación simbiótica, ha sido realizada parcialmentc. Esto se traduce, en una imperiosa necesidad de disponer de un catastro actualizado, que 
permita establecer la asociación y de esta forma, ser utilizado para identificar raices en estado funcional (Agerer, 1987- 1995; 1991; 1995).

El objetivo del presente estudio es, contribuir al catastro de ectomicorrizas en especies nativas de Chile, mediante la caracterización morfo-anatomía de la micorriza: Laccaria laccata (Skop.) Berk + Nothofagus dombeyi (Mirb.) Oerst. y Paxillus involutus (Batsch) Fr. + Nothofagus alpina. (P. et. E.,) Oerst.

\section{MATERIALES Y METODOS}

El estudio fue realizado con material colectado en el vivero VIFOREM, ubicado en la Provincia de Valdivia, Sur de Chile ( $39^{\circ} 75^{\prime}$ L.S.).

La nomenclatura de los hongos simbiontes fue de acuerdo a Garrido (1988) y Valenzucla (1993) y para los hospedantes, según Marticorena \& Quezada (1985). Los cuerpos fructiferos de las especies de hongos, fueron conservados para su detcrminación en estado fresco y posteriormente secados, para mantenerlos en la colección del Herbario Botanische Staatssamlung Münich, Alemania ( $M$ ).

Para la caracterización morfo-anatómica, el material radical fue colectado de acuerdo a la metódica de Agerer (1991) y observado a microscópía estercoscópica, para la caracterización del hábito de la micorriza. Para el estudio de la micorriza y su fotomicroscopía. se emplearon secciones transversales y longitudinales, scgún la metódica indicada en Agerer (1991, 1995.). La caracterización del manto fúngico fue realizada mediante test químicos (Agerer,1987-1995) y las preparacioncs microscópicas fueron depositadas en el Herbario Botanische Staatssamlung Münich (M).

\section{RESULTADOS Y DISCUSION}

\author{
Laccaria laccata (Scop.) Berk + Nothofagus dombeyi
} (Mirb.) Oerst.

Colecta: Vivero Viforem, Pelchuquín Prov. de Valdivia, 2.05.1995 (GP1901)

\section{Descripción de la ectomicorriza:}

\section{Hábito:}

El hábito de la micorriza cs monopodial, tortuosa, irregularmente pinnada y posee una longitud variable entre $0.8-1.5 \mathrm{~cm}$ con un diámetro que oscila entre 0.2 $0.4 \mathrm{~mm}$ (Fig. 1.a). Ramificaciones dicotómicas se han observado en coníferas (Mohan etal. 1993). La coloración es blanca, de aspecto levemente algodonosa, particularmente en los estadios juveniles, mientras que en cdad más avanzada, posee una coloración cafe claro, características observadas para otras especics (Agerer 1987-1995, Mohan et al. 1993)

\section{Manto:}

La superficie del manto es levemente pubescente, con hifas emanantes tortuosas, de tamaños que alcanzan hasta $150 \mathrm{um}$. Las células corticales de la raíz no son visibles. Hifas rizomorfas frecuentes, de color blanco con ramificaciones irregularmente dicotómicas. La estructura del manto presenta un espesor de 10-50 $\mu \mathrm{m}$. Una vista superficial del manto con aspecto plectenquimatoso, detaIla la presencia de septos a menudo con fibulas (Fig. 2a). Observaciones del manto en las capas internas, indican una estructura plectenquimatosa más compacta, hasta constituir un pseudoparénquima de hifas cortas. Presencia de septos ocasionalmente con fíbulas(Fig. 2 b). Estos caracteres del manto han sido descritos para L. laccata en Pinus patula, destacando que, en estadios ontogénicos iniciales el desarrollo de la micorriza se asemeja a Telephora terrestris (Mohan et al. 1993).

\section{Sección longitudinal:}

Una sección longitudinal de la micorriza (Fig. 2 c), destaca la secuencia desde el exterior, que incluye el manto y células corticales con hifas intercelulares (red de Hartig), que evidencian una inclinación hacia el ápice de la micorriza. Se observan varios estratos corticales sin infección, los cuales se suceden topográficamente, con la endodermis y cilindro central.

\section{Sección transversal:}

La micorriza, destaca en sección transversal el manto fúngico con tamaño variable entre 10-50 um (Fig. 2d). con la red de Hartig claramente definida, conformada por células hifas ovales, hasta globosas. En la ilustración se advierte, células parenquimatosas de la corteza, endodermis y cilindro central del hospedante.

\section{Paxillus involutus (Batsch) Fr. + Nothofagus alpina (P. et E.) Oerst. \\ Colecta: Vivero VIFOREM, Pelchuquín Prov. de Valdivia.2.05.1995. (GP 1902)}

\section{Descripción de la ectomicorriza}

\section{Hábito:}

Mucstras de raices finas presentaron una micorriza regularmente monopodial, con tamaño variable de hasta $15 \mathrm{~mm}$, diámetro que fluctua entre $0,3-0,4 \mathrm{~mm}$, con algunas ramas latcrales cortas de hasta $3 \mathrm{~mm}$. Extremos de las ramas. con terminaciones agudas y' con algunas ramas laterales cortas (Fig. 1 b). Presencia de hifas emanantes y rizomorfas. Los esclerocios son frecuentes de observar y presentan forma esférica. de hasta $1 \mathrm{~mm}$ de diámetro, de carácter pscudoparenquimatoso, con células 
angulares. (Fig. 1.b). La coloración de la micorriza es café-amarillenta clara en estadios juveniles, para cambiar a un color café más oscuro, en etapa adulta. Esta caracterización es coincidente con los aportes realizados por Brun et al. (1995), para los estadios ontogenéticos iniciales, de la simbiosis con Betula pendula.

\section{Manto:}

El manto posee un diámetro variable que fluctua entre 20-50um, presentando en vista superficial, una estructura plectenquimatosa laxa. A menudo se presentan septos con fibulas y se distinguen doliporos y anastomosis. (Fig. 3a). Una vista interior del manto, refleja un tejido plectenquimatoso más compacto, con hifas cortas y septos a veces con fibulas (Fig. 3b). Superficie de los ápices micorrizados, con un mantodensamente plectenquimatoso.

\section{Hifas radiantes y rizomorfas:}

| Se observan con frecuencia en la micorriza hifas radiantes ramificadas. Un detalle de hifas rizomorfas indican una longitud de 3-5,5 mm. En una visión longitudinal de las rizomorfas, que en aigunos casos presentan ramificaciones dicótomas irregulares, se presentan hifas centrales y septos con algunas fibulas (Fig. 3c). El diámetro de las rizomorfas puede alcanzar hasta $40 \mu \mathrm{m}$, con una coloración café claro.

\section{Sección longitudinal:}

En esteplano, es posible observar que las micorrizas de los primeros estadios de desarrollo, presentan células del hongo que envuelven las capas de la epidermis, formando una red de Hartig periepidérmica, carácter ya señalado por Agerer (1991) y Brun et al. (1995), para la misma especie de hongo. Se observa además que los estratos internos del manto aparecen formados por un tejido más compacto, plecténquimatoso, hifas con presencia de septos y fíbulas.

\section{Sección transversal:}

La micorriza en sección transversal (Fig.3d), detalla el aspecto del manto y la presencia de taninos en la corteza parenquimática de la raiz. En la zona de infección (red de
Hartig), es posible identificar lóbulos de hifas (tipo palmetti), observadas de igual forma en la micorriza Cenococcum geophilum $\mathrm{Fr}+$ Nothofagus alessandrii Esp (Flores et al. 1997)

\section{CONCLUSIONES}

- La descripción y caracterización morfo-anatómica de las ectomicorrizas en $N$. alpina y $N$. dombeyi, contribuyen a aumentar el catastro para las estomicorrizas en las especies nativas de Chile, constituyendo uno de los primeras descripciones para especies del gén. Nothofagus, en el Cono Sur de América.

- Las especies de Paxillus involutus y Laccaria laccata, corresponden a especies altamente promisorias para ensayos de inoculación en vivero, pues colonizan con alta frecuencia los estadios de plántulas para $N$. alpina y $N$. dombeyi, respectivamente.

- Por la importancia que presentan las asociaciones micorrízicas para las especies de Nothofagus, particularmente en el estado de plántulas, la aplicación de inóculo micorrízico en la fase de viverización, contribuirá a fortalecer los programas de reforestación.

-Un catastro actualizado de las descripciones morfoanatómicas, contribuirá a identificar a los componentes de la asociación y a explicar las relaciones fisiológicas y mecanismos de reconocimiento entre los simbiontes, aspecto de vital importancia en el establecimiento y éxito de los programas de reforestación, con especies nativas en el Sur de Chile.

\section{AGRADECIMIENTOS:}

El estudio fue desarrollado con el apoyo del Proyecto Fondecyt 1940849 y 1970707.

G. Palfner, agradece la beca otorgada por la Konrad Adenauer Stiftung.

\section{REFERENCIAS}

Agerer, R. (1987-1995). Atlas of ectomycorrhizae. Einhon Verlag Germany

- (1991). Characterization of ectonycorrhizae. In: Read, D.J, \& Varma, A. \& Norris, J. R. (Eds.). Techniques for the study of mycorrhiza. Academic Press, London. Methods in Microbiology 23: 25-73

- (1995). Anatomical characteristics of identifiedectomycorrhizae.
An attempt towards a natural classificaction. In: Hock, B. \& Varma, A.K. (Eds.). Mycorrhiza: structura, function and molecular biology an biotechnology. Berlin, Germany, 685-734

Brun, A.; Chalot, M.; Finlay, R.D.; Söderstrom, B. (1995). Structure and function of the ectomycorrhizal association between Paxillus involutus (Batsch) Fr. and Betula pendula Roth. New Phytol. 129: 487-493 

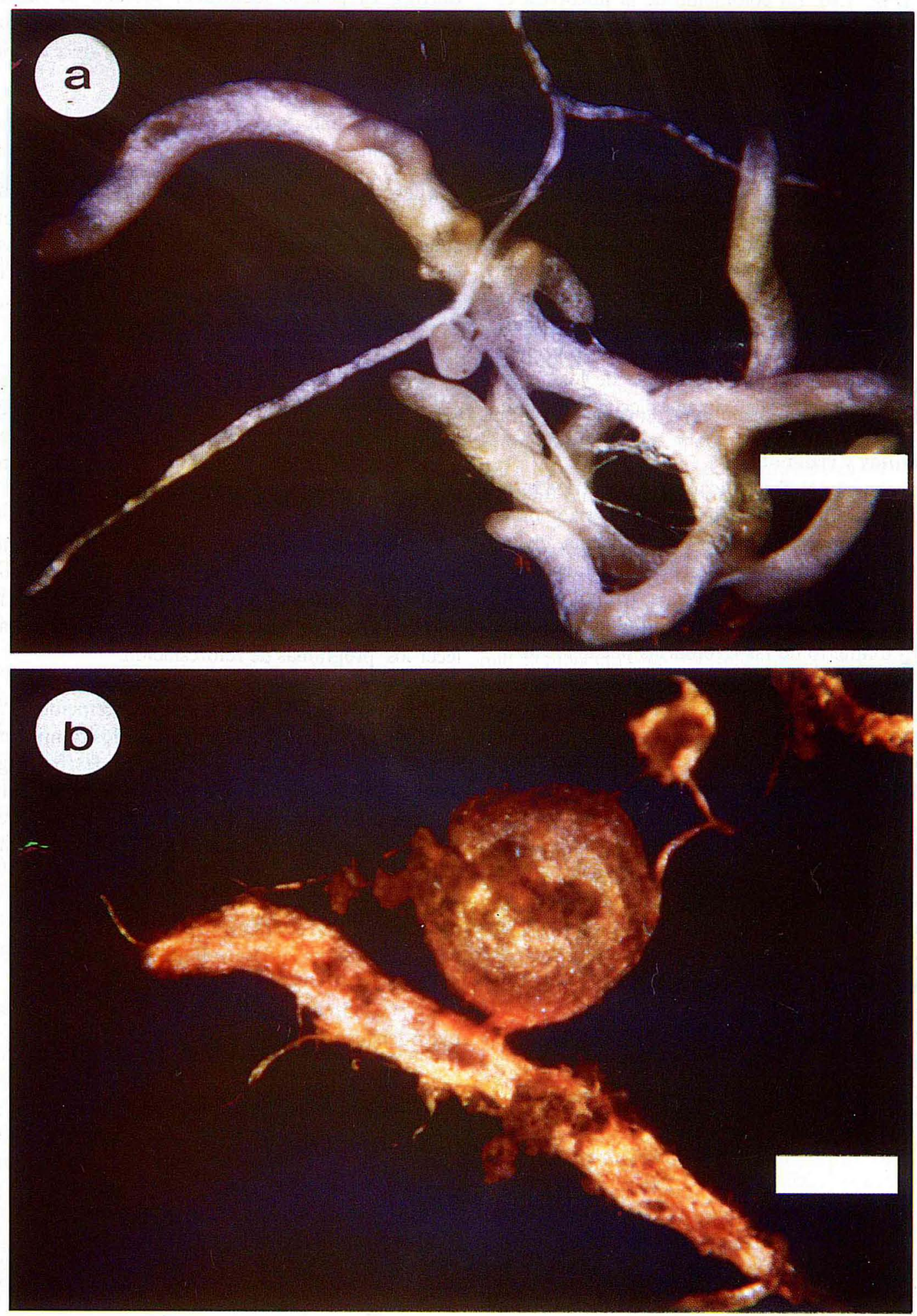

Fig. 1.- a: Laccaria laccata (Scop.) Berk+ Nothofagus dombeyi (Mirb.) Oerst. Hábito de la ectomicorriza (escala= $5 \mathrm{~mm}$ ). b: Paxillus involutus (Batsch.) Fr. + Nothofagus alpina (P. et E.) Oerst. Hábito de la ectomicorriza con esclerocios $($ escala $=5 \mathrm{~mm})$. 

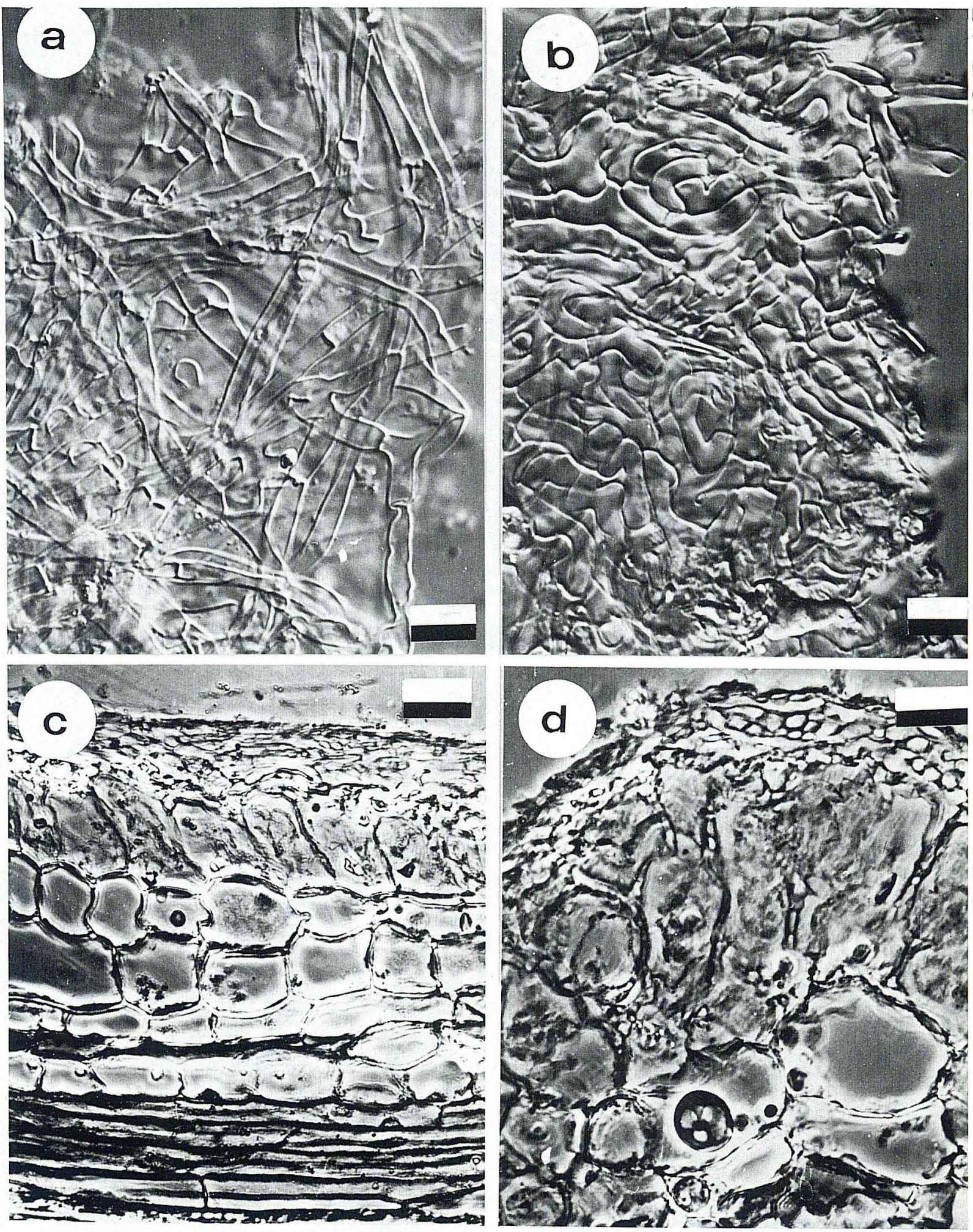

Fig. 2.- Ectomicorriza de Laccaria laccata (Scop) Berk+ Nothofagus dombeyi (Mirb.) Oerst. a: capa superficial del manto, plecténquima de largas hifas y septos con fibulas (escala $=10 \mu \mathrm{m}$ ), b: capa interior del manto fúngico, hifas cortas (escala: $10 \mu \mathrm{m}$ ), c: sección longitudinal con cilindro central, endodermis, corteza, hifas intracelulares y manto fúngico (escala $25 \mu \mathrm{m}$ ), d: sección transversal, manto fúngico, red de Hartig, endodermis y cilindro central. 


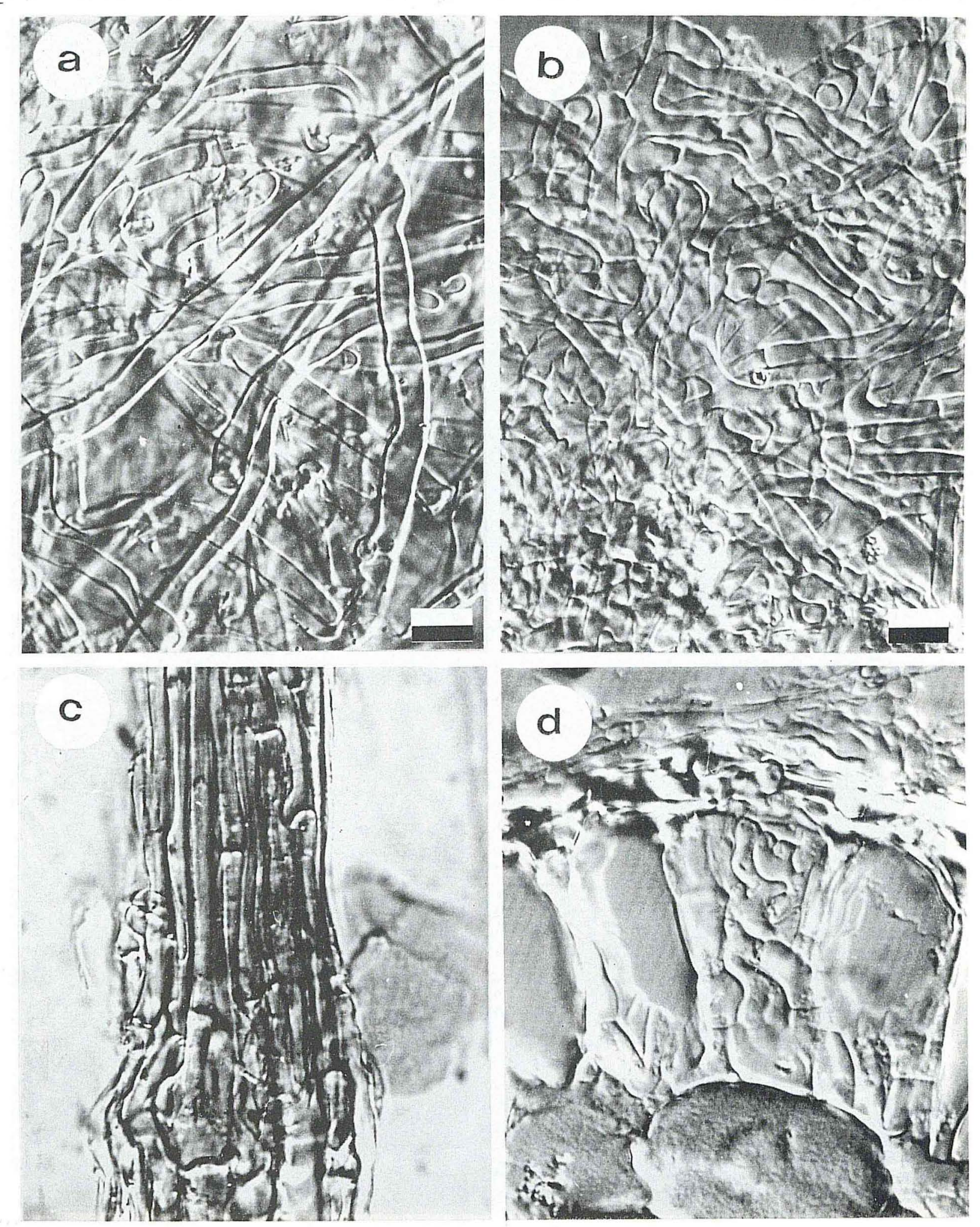

Fig. 3.- Ectomicorriza de Paxillus involutus (Batsch.) Fr. + Nothofagus alpina (P. et. E.) Oerst. a: vista superficial del manto plecténquimático (escala : $10 \mu \mathrm{m}$ ), b: vista interior del manto plectenquimático (escala : $10 \mu \mathrm{m}$ ), c: Rizomorfa en vista longitudinal (escala $10 \mu \mathrm{m}$ ), d: sección transversal de micorriza, red de Hartig con lóbulos de hifas (escala: $10 \mu \mathrm{m})$. 
Flores, R.;Godoy, R.;Palfner, G. (1997). Morfo-inatomia de la ectomicorriza Cenococcum geophilum Fr en Nothofagus alessan-drii Esp. Gayana (Botánica) (en prensa)

Gamundi, I. \& Horak, E (1993). Hongos de los bosyues andino-patagónicos. Guia para el reconocimiento de las especies más comunes. Vazquez Mazzini Editores, Argentina

Garrido, N. (1988). Agaricales und ihre Mykorrhizen in den NothofagusWäldern Mittelchiles. Biblotheca Mycologica 120:1-528

Godoy, R.; Carrillo, R.;Peredo, H. (1995). Ökologische und experimentelle arbeiten über Mykorrhiza in Naturwäldern Südchiles. Verh. Gess. f. Ökol 24:619-622

Kalin-Arroyo, M.; Armesto, J.;Donoso, C.;Murua, R.; Pisano,E.; Schalatter, R.; Serey, I. (1995). Hacia un provecto forestal ecológicamente sustentable. Rev. Chil. Hist. Natural 68:529-538

Marticorena, C. \& Quezada, M. (1985). Catalogo de la flora vaseular de Chile. Gayana (Botánica) : 1-105

Marx, D. (1991). The practical significance of ectomycorrhizae in forest establischment. In: Ecophysiology of ectomycorrhizae of forest tree. The Marcus Wallenberg Foundation, Symposium Procc., 7:54-90
Mohan, V.; Natarajan, K.; Ingleby, K. (1993). Anatomical studies on ectomycorrhizas II. The ectomycorrhizas produced by Amanita muscaria, Laccaria laccata and Suillus bresipes on Pinus patula. Mycorrhiza $3: 43-49$

Palfner, G. \& Godoy, R. (1996 a). «Nothofagirhiza vinicolor» Nothofagus pumilio (Poeppp. et Endl.) Krasser. Descriptions of Ectomycorrhizae, 1:65-70 .(1996 b). Russula fueguiana Singer Nothofagus pumilio (Poepp. et Endl.) Krasser. Descriptions of Ectomycorrhizae, 1: $1321-136$

Perry, D. \& Amaranthus, M. (1990). The land-soil bootstrap: microorganisms and reclamation of degraded ecosystems. In J.J. Berger (ed.). Envirionment al restoration the Earth, Island Press, Washington D.C., pp.94-102

Singer, R. \& Morello, J. (1960). Ectotrophic forest tree mycorrhizae and forest communities. Ecology 41:549-551

Valenzucla, E. (1993). Estudio sistemático, corológico y ecológico de los Agaricales Sensu Lato de los bosques autóctonos de la región de los Lagos en Chile. Tesis Doctoral, Universidad de Alcalá de Henares, España. 